\title{
The Effect of Extraction Conditions and Film Side on the Molecular Conformation of Silk Sericin Film
}

\author{
Yoon Nam Jo, Do Gyu Bae, and In Chul Um* \\ Department of Bio-fibers and Materials Science, Kyungpook National University, Daegu 702-701, Republic of Korea
}

\section{Abstract}

In this study, silk sericin films were prepared using different extraction methods, and the molecular conformation of sericin was examined using Fourier-transform infrared spectroscopy with attenuated total reflection geometry. Additionally, the effect of the film side (air-facing side or plate-facing side) on the molecular conformation of the sericin films was investigated. Interestingly, the molecular conformation of the sericin film depended on the film side. The molecular conformation of air-facing side of the sericin film was significantly influenced by extraction solution and time. The $\beta$-sheet crystallization and the crystallinity index of the sericin film markedly increased with an increase in the extraction time in hot water. The order of the crystallinity indices for the sericin films obtained with different extraction solutions was as follows: citric acid solution > urea solution >> hot water. In contrast, no remarkable differences were observed in the molecular conformation of the plate-facing side of the sericin film after extraction in hot water for different time periods. Urea and citric acid solution extractions showed remarkably higher crystallinity indices for sericin than those obtained after hot water extraction. However, no significant differences were observed in the crystallinity index of sericin between urea and citric acid solution extraction in plate-facing side of the film.

Received : 22 May 2013

Accepted : 29 May 2013

Keywords:

Silk sericin,

Extraction method,

Molecular conformation,

Film side,

Crystallinity index

\section{Introduction}

Silk is a naturally occurring material from silkworms and consists of fibroin and sericin. Silk has been used as a high quality textile material for a long time. Sericin is usually removed from silk through a degumming process that significantly improves the luster and touch feeling of silk fiber. Considering that silk contains about $25 \%$ sericin, a high amount of sericin is lost through the degumming process because most people believe that sericin is not a useful material. However, recent studies have focused on determining the useful and valuable properties of sericin, and the results of these studies have established many unique and valuable properties of sericin as a biomaterial.

Tsubouchi et al. (2005) reported that sericin enhances the attachment of cultured human skin fibroblasts. Nagai et al. (2009) reported that sericin promotes wound healing and wound size reduction. Aramwit et al. (2009) monitored inflammatory mediators induced by silk sericin and concluded that sericin promotes the wound healing process without causing

\section{*Corresponding Author :}

In Chul Um

Department of Bio-fibers and Materials Science, Kyungpook National University, Daegu 702-701, Republic of Korea Tel: +82-53-950-7757 / FAX: +82-53-950-6744

E-mail: icum@knu.ac.kr

(C) 2013 The Korean Society of Sericultural Sciences 
inflammation. Limpeanchob et al. (2010) reported that sericin reduces serum cholesterol levels in rats and cholesterol uptake into Caco-2 cells. Seo et al. (2011) reported that the cholesterol and blood glucose lowering effect of silk was enhanced by increasing the sericin content. Dash et al. (2008) reported that silk sericin from the tasar silkworm inhibits ultraviolet B (UVB)induced apoptosis in human skin keratinocytes. These findings indicate that silk sericin is an important biomaterial in the cosmetic, functional health food, and biomedical industries.

In addition to these applied studies, many studies into the extraction of sericin from silk have also been performed to obtain sericin with a higher molecular weight (MW) or better functional properties. It is generally accepted that molecular degradation of sericin occurs during hot water extraction and that degradation of sericin during extraction depends on the extraction method. To date, the most effective extraction method for obtaining highMW sericin was to extract sericin using $8 \mathrm{M}$ urea solution with 5\% 2-mercaptoethanol (Takasu et al., 2002). However, this method is extremely tedious and expensive. Therefore, Oh et al. (2011) extracted sericin from silk using hot water and precipitated it using ethanol to obtain sericin with a high MW. In addition, they and coworkers studied the extraction conditions of Antherae mylitta sericin with high yields and MW and reported that $0.02 \mathrm{M}$ sodium carbonate and boiling for $60 \mathrm{~min}$ were the optimum conditions (Yun et al., 2013). Aramwit et al. (2010) investigated the effect of the extraction method on the cell viability of sericin and reported that sericin cell viability and collagen production depended on the extraction method of sericin. These studies indicate that the extraction method for sericin strongly affects the MW as well as the biological properties of sericin.

The molecular conformation of silk protein has been studied using Fourier-transform infrared (FTIR) spectroscopy because it plays an important role in determining the properties of silk protein. Kim et al. (2012) examined the molecular conformational changes of silk sericin in the presence of lyoprotectant using FTIR spectroscopy. Kweon et al. (2011) investigated the solubility and molecular conformation of silk fibroin membrane using FTIR spectroscopy to determine its use as an eardrum patch. Kim et al. (2013) utilized the crystallinity index calculated from random coil and $\beta$-sheet conformations to examine the homogeneity of silk degumming. Um et al. (2001) compared the molecular conformation of the silk fibroin films obtained using different preparation methods, and they showed that $\beta$-sheet crystallization of silk fibroin was induced by formic acid during the evaporation of formic acid using FTIR spectroscopy (Um et al. 2003).

In this study, we extracted sericin using various extraction solutions and times and prepared its film. We examined the effect of the extraction method on the molecular conformation of the sericin film. Additionally, we examined the effect of the film side (air-facing or petri-dish plate-facing) on the molecular conformation of the sericin film using an FTIR spectrometer with attenuated total reflection (ATR) geometry.

\section{Materials and Methods}

\section{Preparation of sericin film}

Baekokjam silkworm cocoons were used to extract silk sericin. Silk sericin was extracted using 3 different extraction solutions: distilled water, citric acid solution, and urea solution. For the water extraction, sericin was extracted by immersing the cocoons in water at $100^{\circ} \mathrm{C}$ for $30 \sim 120 \mathrm{~min}$. For the citric acid extraction method, the cocoons were treated in a $1.25 \%(\mathrm{w} / \mathrm{v})$ aqueous citric acid solution at $100^{\circ} \mathrm{C}$ for $30 \mathrm{~min}$. The extracted sericin solution was dialyzed in distilled water using a dialysis tube (MW cutoff $=12,000 \sim 14,000)$ for 7 days to remove the citric acid. For the urea extraction, the cocoons were treated in an $8 \mathrm{M}$ aqueous urea solution at $85^{\circ} \mathrm{C}$ for $30 \mathrm{~min}$ after immersion in the same solution at room temperature for $30 \mathrm{~min}$. The extracted sericin solution was dialyzed to eliminate urea using the same method as that used in citric acid extraction. All extracted sericin solutions were filtered into non-woven fabric twice before dialysis (for acid and urea extraction) or drying (hot water extraction). Aqueous sericin solutions from the 3 different extraction methods were poured into petri-dishes and dried at $40^{\circ} \mathrm{C}$ in a drying oven to form the sericin film.

\section{FTIR measurements and determination of the crystallinity index}

The FTIR (Nicolet 380; Thermo Fisher Scientific, USA) spectra of the sericin films were obtained using the ATR method. To examine the effect of the sericin film side, 2 different sides (air-facing and plate-facing) of the sericin film (Fig. 1) were measured using ATR geometry. The crystallinity index was 


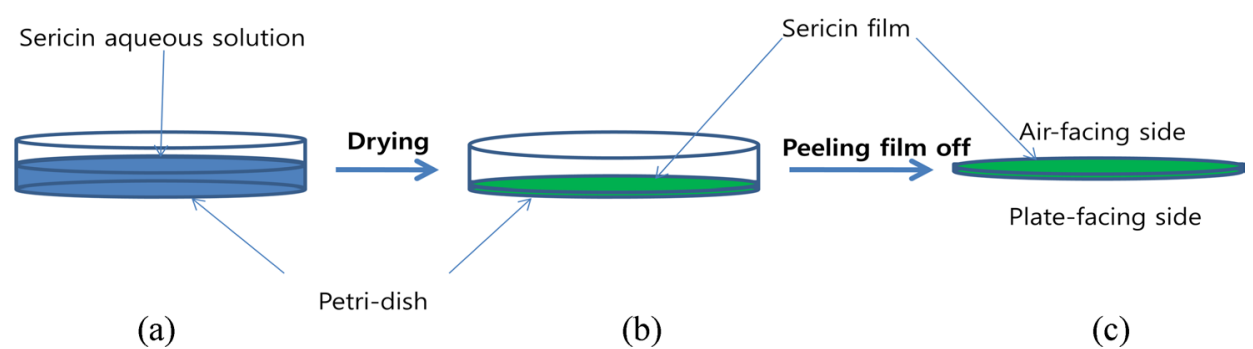

Fig. 1. Schematic drawing for the sericin film casting process and the 2 different sides (air-facing and plate-facing) of the sericin film; (a) aqueous sericin solution in a petri-dish, (b) dried sericin film in a petri-dish, and (c) sericin film peeled off from a petri-dish.

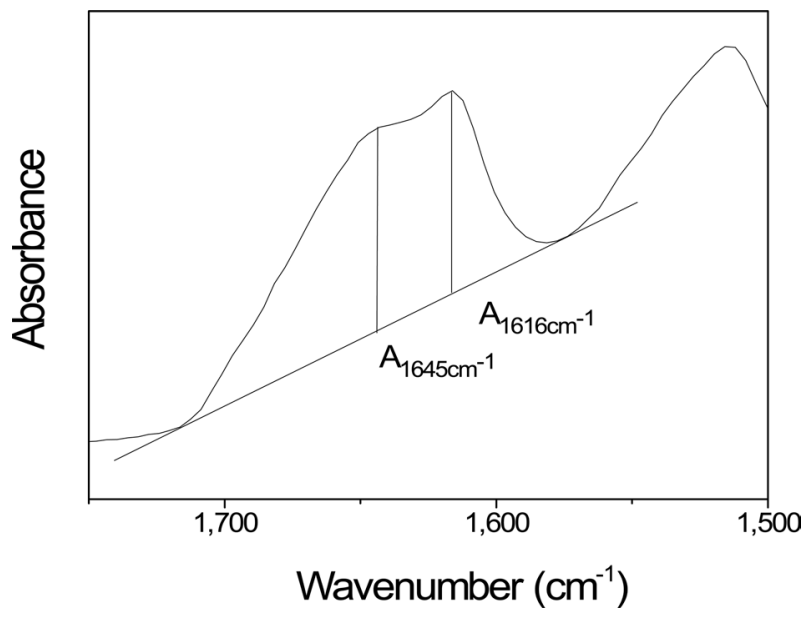

Fig. 2. Determination of the crystallinity index from the amide I band.

calculated using an absorbance of 1616 and $1645 \mathrm{~cm}^{-1}$ from the FTIR spectrum, which corresponded to the $\beta$-sheet and random coil conformations, respectively (Fig. 2). The crystallinity index was calculated using the following equation:

Crystallinity index $(\%)=\frac{\mathrm{A}_{1616 \mathrm{~cm}^{-1}}}{\mathrm{~A}_{1616 \mathrm{~cm}^{-1}}+\mathrm{A}_{1645 \mathrm{~cm}^{-1}}} \times 100$

$\mathrm{A}_{1616 \mathrm{~cm}}{ }^{-1}$, Absorbance at $1616 \mathrm{~cm}^{-1}$

$\mathrm{A}_{1645 \mathrm{~cm}^{-1}}$, Absorbance at $1645 \mathrm{~cm}^{-1}$

To obtain the average and variations of the crystallinity index, FTIR measurements were performed on 7 different parts of the sericin film.

\section{Results and Discussion}

The FTIR spectra of the air-facing side of the sericin film prepared using hot water extraction with different extraction

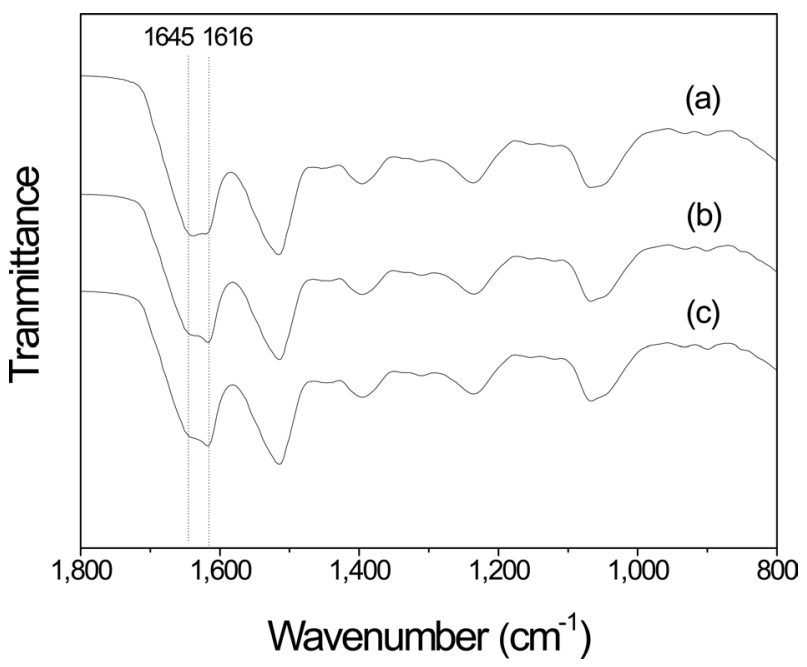

Fig. 3. Fourier-transform infrared (FTIR) spectra for the air-facing side of the silk sericin film with hot water extraction for different time periods: (a) $30 \mathrm{~min}$, (b) $60 \mathrm{~min}$, and (c) $120 \mathrm{~min}$.

times are shown in Fig. 3. After extraction for $30 \mathrm{~min}, 2$ overlapping peaks at 1645 and $1616 \mathrm{~cm}^{-1}$ in amide I band, which corresponded to a random coil and $\beta$-sheet conformation (Um et al., 2001; Kim et al., 2013), respectively, were observed. With an increase in the extraction time, the IR absorption peak at 1616 $\mathrm{cm}^{-1}$ became stronger and that at $1645 \mathrm{~cm}^{-1}$ became weaker. This indicates that the content of the $\beta$-sheet crystallite of sericin increases with an increase in the extraction time in hot water.

The FTIR results of the plate-facing side (petri-dish facing side) of sericin film were different from those of the airfacing side of film. After extraction for $30 \mathrm{~min}$ (Fig. 4), two IR absorption peaks were observed at 1645 and $1616 \mathrm{~cm}^{-1}$; the latter peak slightly stronger than the former. However, unlike the FITR spectra of the air-facing side of the sericin film, that of the platefacing side of the sericin film did not change with a change in the extraction time. 


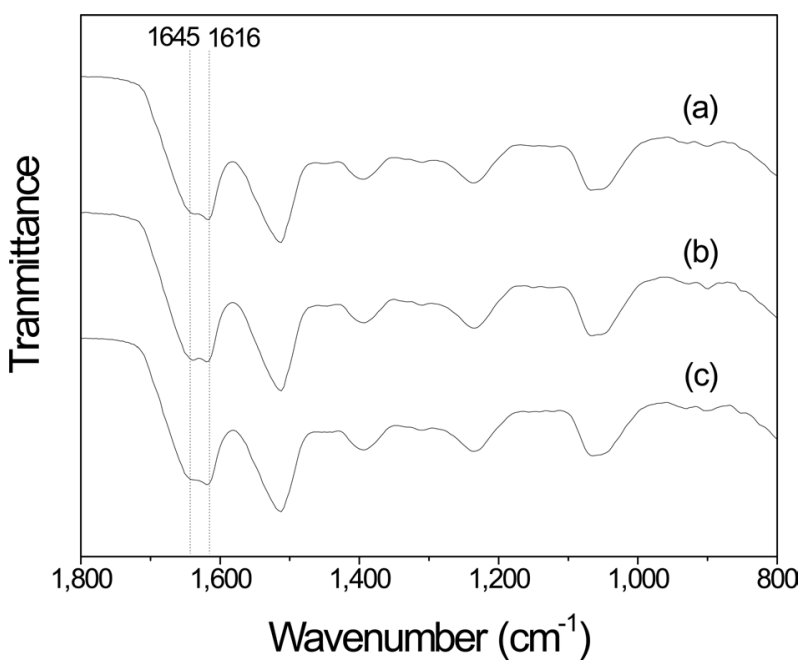

Fig. 4. Fourier-transform infrared (FTIR) spectra for the plate-facing side of the silk sericin film after hot water extraction times for different time periods: (a) $30 \mathrm{~min}$, (b) $60 \mathrm{~min}$, and (c) $120 \mathrm{~min}$.

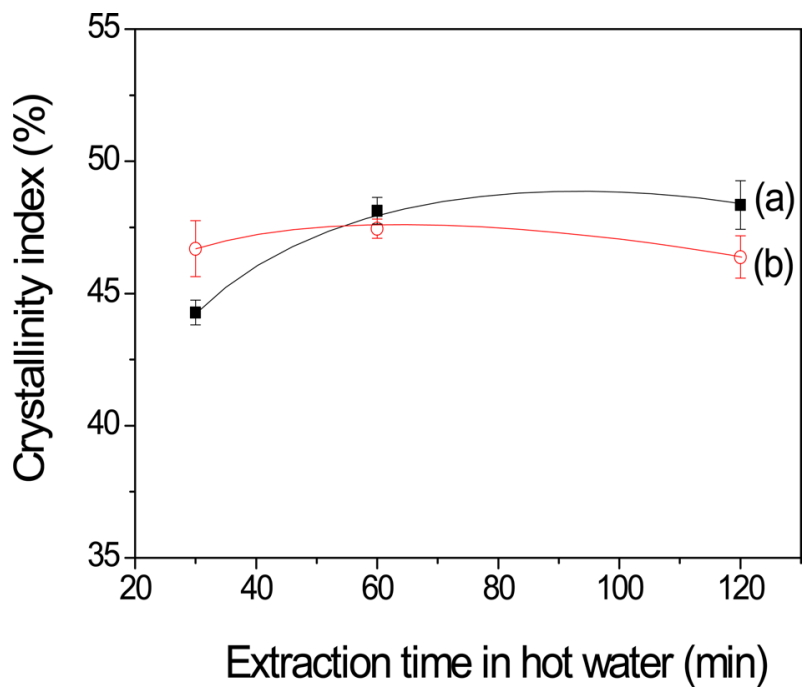

Fig. 5. Effect of the film side on the crystallinity index of silk sericin film after hot water extraction for different time periods; (a) airfacing side and (b) plate-facing side.

The crystallinity index calculated from amide I band (Fig. 5) clearly indicates different trend of crystallinity of the sericin film between the air-facing and the plate-facing sides. The crystallinity index of the air-facing side of the silk sericin film prepared by extraction for 30 min was $44.3 \%$. The crystallinity index increased up to $48.3 \%$ when the extraction time increased to $120 \mathrm{~min}$. In contrast, for the plate-facing side of the sericin film, the crystallinity index ranged from 46 to $48 \%$, and it did not almost change with a change in the extraction time.

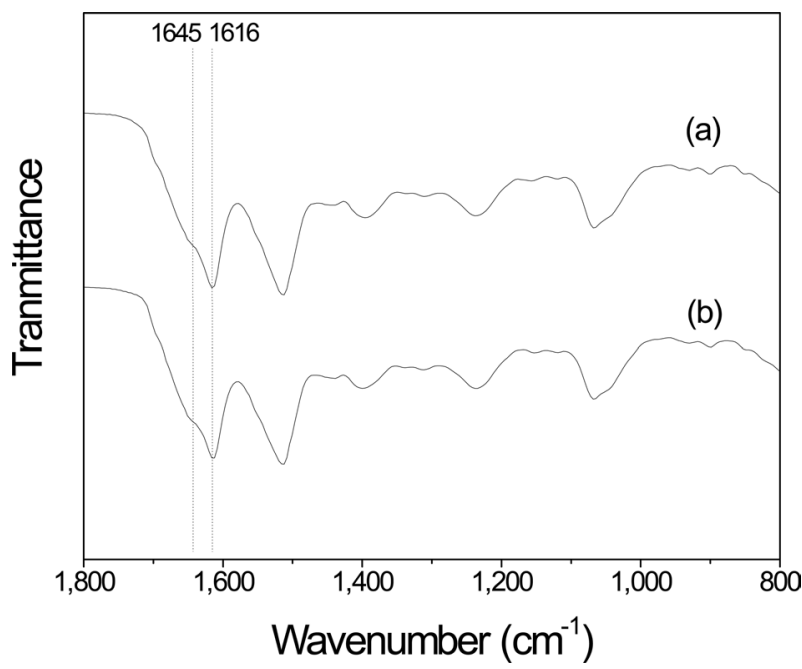

Fig. 6. Fourier-transform infrared (FTIR) spectra for the air-facing side of the silk sericin film with different extraction solvents: (a) aqueous citric acid solution and (b) aqueous urea solution.

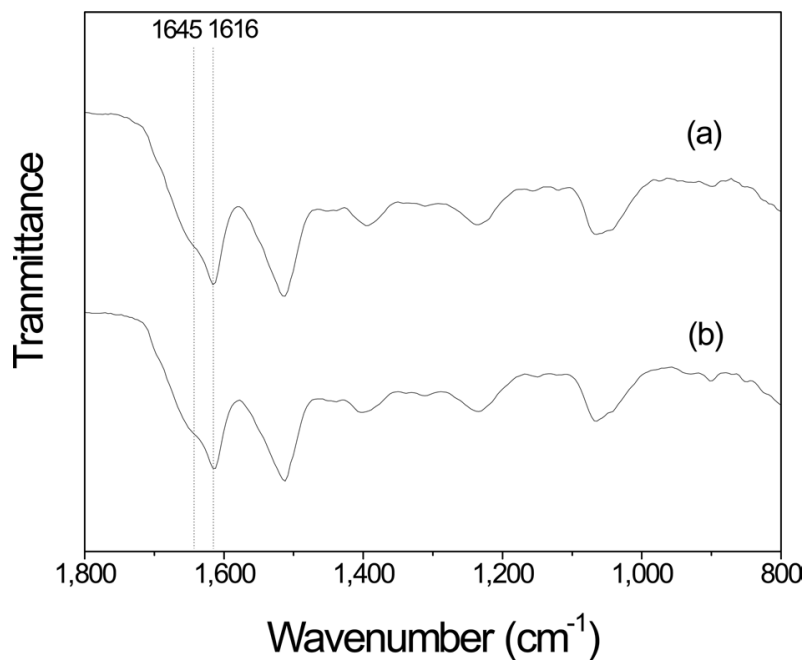

Fig. 7. Fourier-transform infrared (FTIR) spectra for the plate-facing surface of the silk sericin film with different extraction solvents: (a) aqueous citric acid solution and (b) aqueous urea solution.

The FTIR spectra of the air-facing side of the sericin films prepared by extracting sericin in an aqueous citric acid solution and an aqueous urea solution are shown in Fig. 6. The two sericin films showed a strong IR absorption peak at $1616 \mathrm{~cm}^{-1}$ with a weak shoulder peak at $1645 \mathrm{~cm}^{-1}$. This indicates that these films contained a high amount of $\beta$-sheet crystallite. The FTIR spectra of the plate-facing side of the sericin films extracted using the two solutions is shown in Fig. 7. The FTIR spectra of these films were similar to those of the air-facing side of the 


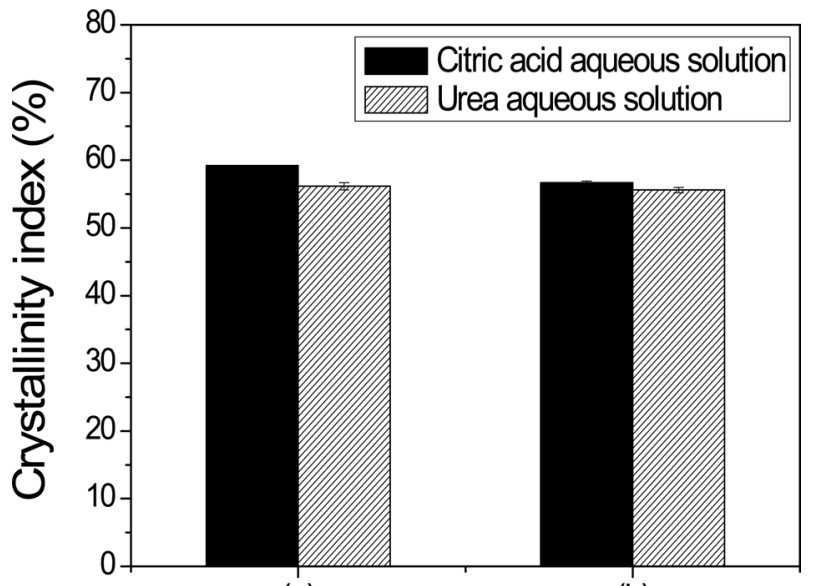

(a)

(b)

\section{Extraction solution}

Fig. 8. Effect of the film side on the crystallinity index of the silk sericin film extracted with an aqueous citric acid solution and aqueous urea solution: (a) air-facing side and (b) plate-facing side.

sericin films. However, the trend of crystallinity index of the airfacing side of the film was different from that of the plate-facing side of the film (Fig. 8). The crystallinity index of air-facing side of the sericin film extracted from an aqueous citric acid solution was higher than that of the film obtained from an aqueous urea solution by $2.5 \%$ (Fig. 8 (a)). However, the crystallinity indices of the plate-facing side of two sericin films extracted using different extraction solutions were not significantly different.

The results of the FTIR studies on the sericin films for the different film side (air- or plate-facing) following different extraction conditions (extraction time in hot water or extraction solvent) showed several interesting features.

Firstly, the film side (air-facing or plate-facing) showed different trends of molecular conformation and crystallinity indices of the silk sericin films. In the case of air-facing surface of the film, the molecular conformation of sericin was significantly influenced by (1) the extraction time in hot water and (2) the type of extraction solution. However, no remarkable differences were observed for the plate-facing surface of the sericin film in terms of the above two factors. Although the exact mechanism underlying this behavior cannot be explained in this study, our findings indicate that different crystallizations of sericin take place on the air-facing surface and on the bulk side (or inside) of the sericin solution.

A second interesting feature is the increase of the crystallinity index for the air-facing sericin film surface following increased extraction time. It seems that this is related to the MW of sericin. That is, the hydrolytic molecular degradation of sericin takes place during the extraction of sericin in hot water. Therefore, the MW of sericin decreases with an increase in the extraction time of sericin in hot water. This indicates that the crystallinity of sericin increases when the MW of sericin decreases. This may be because of the difference of molecular mobility between sericins of different MWs. That is, as the MW of sericin decreases, the molecular mobility increases. The sericin molecules can arrange and crystallize more easily when their mobility is increased. In contrast, the lower molecular mobility of the higher MW of sericin might restrict the molecular arrangement of sericin resulting in a lower level of $\beta$-sheet crystallite formation.

Lastly, the crystallinity indices of the sericins extracted with the aqueous citric acid solution and aqueous urea solutions were much higher than those for the sericin films obtained through hot water extraction. It is thought that this result is intimately related to the different preparation methods of the sericin film and the gelation behavior of sericin. That is, the sericins extracted from hot water were prepared by casting aqueous sericin solutions in petri-dishes just after the hot water-extracted sericins were added. In contrast, the sericin films extracted with the aqueous citric acid and urea solutions were dialyzed to remove the citric acid and urea respectively. Thus, after 7 days dialysis, aqueous sericin solutions are obtained and the films are prepared by drying the solutions. However, after dialysis, the sericin solutions contain many gels because sericin gels easily and some sericin became gel during the dialysis. When the gelation of sericin takes place, it is well known that $\beta$-sheet crystallization occurs. Therefore, a stronger IR absorption peak and higher crystallinity index were observed for the sericin films extracted with the aqueous citric acid and urea solutions.

It is also interesting to note that the sericin film extracted with the aqueous citric acid solution on the air-facing surface had a higher crystallinity index than when extracted with the urea solution, as can be seen in Fig. 8. This might be due to the different gelation behaviors of sericin between the two extraction methods. The aqueous citric acid solution is acidic because citric acid is an acid. After the extraction of sericin with the aqueous citric acid solution, the $\mathrm{pH}$ of the sericin citric acid solution was lower than the isoelectric point of sericin (i.e. pH 4.0 (Voegeli et al., 1993)). However, the $\mathrm{pH}$ of the aqueous sericin citric acid 
solution passes the isoelectric point and reaches a neutral $\mathrm{pH}$ during dialysis as water has a neutral $\mathrm{pH}$. It has been reported that the gelation of silk protein is accelerated at the isoelectric point because the same amount of $(+)$ and $(-)$ charged silk protein molecules easily bind together resulting in faster gelation and a stronger silk protein gel strength (Hirabayashi et al., 1990; Ayub et al., 1992). Thus, the higher crystallinity index and more $\beta$-sheet crystallization of sericin from the citric acid solution might be due to sericin passing the isoelectric point during dialysis.

\section{Acknowledgement}

This study was supported by the Basic Science Research Program through the National Research Foundation of Korea (NRF) funded by the Ministry of Education, Science and Technology (2012042016).

\section{References}

Aramwit P, Kanokpanont S, De-Eknamkul W, Srichana T (2009) Monitoring of inflammatory mediators induced by silk sericin. J Biosci Bioeng 107, 556-561.

Aramwit P, Kanokpanont S, Nakpheng T, Srichana T (2010) The effect of sericin from various extraction methods on cell viability and collagen production. Int J Mol Sci 11, 2200-2211.

Ayub ZH, Hirabayashi K, Ari M (1992) Effect of $\mathrm{pH}$ on silk fibroin gelation. Sen-I Gakkaishi 48, 141-144.

Dash R, Mandal M, Ghosh SK, Kundu SC (2008) Silk sericin protein of tropical tasar silkworm inhibits UVB-induced apoptosis in human skin keratinocytes. Mol Cell Biochem 311, 111-119.

Hirabayashi K, Ayub ZH, Kume Y (1990) Gelation of silk fibroin. Sen-I Gakkaish 46, 521-524.

Kim HJ, Chung DE, Um IC (2013) Effect of processing conditions on the homogeneity of partially degummed silk evaluated by FTIR spectroscopy. Int J Indust Entomol 25, 54-60.

Kim MK, Kwak HW, Lee JY, Yun H, Kim MH, Lee KH (2012) Effect of lyoprotectant on the solubility and structure and silk sericin. Int J Indust Entomol 25, 133-137.

Kweon H, Kang S-W, Lee K-G, Park KY, Jo YY, Kang P-D, Sung G-B, Lee HS, Yeo JH, Shin KH, Park CH (2011) Solubility and conformation of silk fibroin membrane. Int J Indust Entomol 22, 101105 .

Limpeanchob N, Trisat K, Duangjai A, Tiyaboonchai W, Pongcharoen S, Sutheerawattananonda M (2010) Sericin reduces serum cholesterol in rats and cholesterol uptake into caco-2 cells. J Agric Food Chem 58, 12519-12522.

Nagai N, Murao T, Ito Y, Okamoto N, Sasaki M (2009) Enhancing effects of sericin on corneal wound healing in Otsuka long-evans Tokushima fatty rats as a model of human type 2 Diabetes. Biol Pharm Bull 32, 1594-1599.

Oh H, Lee JY, Kim MK, Um IC, Lee KH (2011) Refining hot-water extracted silk sericin by ethanol-induced precipitation. Int J Biol Macromol 48, 32-37.

Seo C-W, Um IC, Rico CW, Kang MY (2011) Antihyperlipidemic and Body Fat-Lowering Effects of Silk Proteins with Different Fibroin/ Sericin Compositions in Mice Fed with High Fat Diet. J Agric Food Chem 59, 4192

Takasu Y, Yamada H, Tsubouchi K (2002) Extraction and chromatographic analysis of cocoon of the silkworm, Bombyxmori. J Insect Biotechnol Sericol 71, 151-156.

Tsubouchi K, Igarashi Y, Takasu Y, Yamada H (2005) Sericin enhances attachment of cultured human skin fibroblasts. Biosci Biotechnol Biochem 69, 403-405.

Um IC, Kweon HY, Lee KG, Park YH (2003) The role of formic acid in solution stability and crystallization of silk protein polymer. Int J Biol Macromol 33, 203-213

Um IC, Kweon HY, Park YH, Hudson S (2001) Structural characteristics and properties of the regenerated silk fibroin prepared from formic acid. Int J Biol Macromol 29, 91-97.

Voegeli R, Meier J, Blust R (1993) Sericin silk protein: unique structure and properties. Comset Toilet 108, 101-108.

Yun H, Oh H, Kim MK, Kwak HW, Lee JY, Um IC, Vootla SK, Lee KH (2013) Extraction conditions of Antheraea mylitta sericin with high yields and minimum molecular weight degradation. Int J Biol Macromol 52, 59-65. 